\title{
A comparative analysis of the transcriptome profiles of liver and muscle tissue in pigs divergent for feed efficiency
}

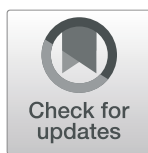

\author{
Stafford Vigors' ${ }^{1}$, John V. O'Doherty ${ }^{2}$, Kenneth Bryan² ${ }^{2}$ and Torres Sweeney ${ }^{*^{*}}$
}

\begin{abstract}
Background: The improvement of feed efficiency is a key economic goal within the pig production industry. The objective of this study was to examine transcriptomic differences in both the liver and muscle of pigs divergent for feed efficiency, thus improving our understanding of the molecular mechanisms influencing feed efficiency and enabling the identification of candidate biomarkers. Residual feed intake (RFI) was calculated for two populations of pigs from two different farms of origin/genotype. The 6 most efficient (LRFI) and 6 least efficient (HRFI) animals from each population were selected for further analysis of Longissimus Dorsi muscle $(n=22)$ and liver $(n=23)$. Transcriptomic data were generated from liver and muscle collected post-slaughter.

Results: The transcriptomic data segregated based on the RFI value of the pig rather than genotype/farm of origin. A total of 6463 genes were identified as being differentially expressed (DE) in muscle, while 964 genes were identified as being DE in liver. Genes that were commonly DE between muscle and liver $(n=526)$ were used for the multi-tissue analysis. These 526 genes were associated with protein targeting to membrane, extracellular matrix organisation and immune function. In the muscle-only analysis, genes associated with RNA processing, protein synthesis and energy metabolism were down regulated in the LRFI animals while in the liver-only analysis, genes associated with cell signalling and lipid homeostasis were up regulated in the LRFI animals.

Conclusions: Differences in the transcriptome segregated on pig RFI value rather than the genotype/farm of origin. Multi-tissue analysis identified that genes associated with $\mathrm{GO}$ terms protein targeting to membrane, extracellular matrix organisation and a range of terms relating to immune function were over represented in the differentially expressed genes of both liver and muscle.
\end{abstract}

Keywords: Feed efficiency, Multi-tissue analysis, Pig, Residual feed intake, Transcriptome, RNA-Seq

\section{Background}

Feed is a major economic consideration within the pig industry and accounts for $60-70 \%$ of the total production cost. Strategies which improve feed efficiency are a high priority for the pig industry and aim to reduce feed costs and nutrient excretion. Residual feed intake (RFI) is considered the most appropriate measure with which to examine the biological factors contributing to feed efficiency, as differences in production traits such as body weight, growth rate, body composition and maintenance requirements are accounted for within this measure [1].

\footnotetext{
* Correspondence: torres.sweeney@ucd.ie

'School of Veterinary Medicine, University College Dublin, Belfield, Dublin 4, Ireland

Full list of author information is available at the end of the article
}

Significant efforts have been made in recent years to understand the molecular and physiological basis of RFI in pigs and the findings have highlighted a range of processes underpinning this trait, including nutrient digestion and absorption, protein deposition/turnover, energy metabolism and immune function [2-5]. Several studies have identified changes in mitochondrial function as a key contributor to feed efficiency, aligning to their central role in energy metabolism [6, 7]. On a different theme, variation in the immune responses has also been identified as a factor influencing feed efficiency $[4,8,9]$.

Irrespective of the underlying biological processes contributing to RFI, early life predictors of feed efficiency would be a valuable, as the measurement of individual feed intake is not feasible in commercial production 
systems. In this regard, genomic selection is promising as it would allow for early selection without the requirement for direct measurement of feed intake [10]. Enriching SNP panels for functional and biologically important SNPs would improve the accuracy of genomic predictions in a complex trait such as RFI. Feed efficiency has a moderate heritability $(0.21-0.33)$ therefore, genomic selection should facilitate higher rates of genetic gain compared to the traditional selection methodologies based on pedigree and phenotype [11] and this has been explored in pigs in a number of studies [12-14].

Another targeted mechanism of enriching for functional/predictive SNPs is through the selection of SNPs that alter putative transcription factor binding site motifs in the promoter regions of genes that are differentially expressed (DE) between divergent animals. With this approach, it is vital that transcriptomic analysis is performed in tissues with the greatest influence on RFI such as liver and muscle. Genes identified across multiple metabolically relevant tissues and across different populations of pigs will highlight more robust candidate markers for the trait. The liver and muscle are two key metabolically relevant tissues for RFI $[6,15]$. In light of this, the objective of this study was to compare the liver and muscle transcriptome of pigs divergent in feed efficiency. Two populations of pigs originating from two different genetic lines and breeding farms were used. We hypothesised that genes associated with energy metabolism and immunity will be identified in the liver and muscle which influence feed efficiency in pigs and will be useful candidates for the identification of functional SNPs.

\section{Results}

\section{Performance and feed efficiency}

Differences in feed intake, performance and feed efficiency traits are presented in Table 1 . The animals ranked as LRFI from Farms A and B had a lower RFI value $(-0.14,-0.18$, respectively) compared to the HRFI animals $(0.19,0.20 ; P<0.001)$. The LRFI pigs had lower ADFI than pigs ranked as HRFI from both farms $(P<0.001)$. On average, the LRFI group consumed $340 \mathrm{~g}$ less feed than their HRFI counterparts in from Farm $\mathrm{A}$ and $470 \mathrm{~g}$ in Farm B. The MBW, ADG and final BW did not differ between RFI groups $(P>0.05)$.

\section{Transcriptomic differences between RFI groups}

The numbers of raw reads, trimmed reads, and mapped reads for each sample are in Additional file 1: Table S1. As the pigs were obtained from two different farms of origin that used different sires (genotype), hierarchical clustering was performed to establish the impact of the farm of origin on the transcriptome. The hierarchical clustering was performed for both muscle $(n=22)$ and
Table 1 Characterisation of performance, body composition, intake and efficiency (Least square means and SEM)

\begin{tabular}{lllll}
\hline Trait & Low & High $^{\mathrm{b}}$ & SEM & $P$-value \\
\hline Farm A & 27 & $18^{\mathrm{c}}$ & & \\
RFI & -0.14 & 0.19 & 0.08 & 0.001 \\
ADFI $^{\mathrm{a}}$ & 2.00 & 2.34 & 0.04 & 0.001 \\
MBW & 32.71 & 32.05 & 0.63 & 0.956 \\
ADG & 0.99 & 0.99 & 0.02 & 0.874 \\
FCR & 2.03 & 2.36 & 0.02 & 0.001 \\
BW & 76.62 & 77.70 & 1.36 & 0.833 \\
Farm B & 28 & 35 & & \\
RFI & -0.18 & 0.20 & 0.016 & 0.001 \\
ADFI & 1.87 & 2.34 & 0.05 & 0.0001 \\
MBW & 10.94 & 11.20 & 0.19 & 0.3662 \\
ADG & 0.950 & 1.01 & 0.031 & 0.2112 \\
BW & 90.45 & 96.08 & 2.378 & 0.1086 \\
\hline
\end{tabular}

${ }^{a}$ ADFI Average daily feed intake, MBW Mid-test metabolic body weight, $A D G$ Average daily gain, $R F I$ Residual feed intake, $B W$ Body weight

${ }^{\mathrm{b}} \mathrm{High}=\mathrm{RFI}$ was $>0.5 \mathrm{SD}$ above the mean; medium $=\mathrm{RFI}$ was $\pm 0.5 \mathrm{SD}$ above and below the mean; low $=\mathrm{RFI}$ was $<-0.5 \mathrm{SD}$ below the mean

${ }^{c}$ Number of animals in each RFI group

liver $(n=23)$ tissues. The transcriptome for each tissue generally segregated based on the RFI value (Figs. 1 and 2; HRFI \& LRFI) rather than genotype/farm of origin. Hence, the data will be presented based on RFI status (i.e. LRFI v HRFI). A total of 6463 genes were identified as being DE in muscle, while 964 genes were identified as being DE in liver (Table 2). Of the $6463 \mathrm{DE}$ genes in muscle, 3138 were up regulated while 3325 were down regulated in the LRFI group (Additional file 2: Table S2). Of the over expressed genes, 1722 had a fold change $<1$, 1381 had a fold change between 1 and 2 and 35 had a fold change $>2$. In muscle, the volcano plots (Fig. 3) highlight that the fold changes were relatively small. Of the under expressed genes, 2465 had a fold change $<1,851$ had a fold change between 1 and 2 and 10 had a fold change $>2$.

In the liver, of the $964 \mathrm{DE}$ genes, 467 were up regulated, while 496 were down regulated in the LRFI group (Additional file 3: Table S3. Of the up regulated genes, 365 had a fold change $<1,77$ had a fold change between 1 and 2 and 25 had a fold change $>2$ (Fig. 4). Of the down regulated genes, 384 had a fold change $<1,100$ had a fold change between 1 and 2 and 12 had a fold change $>2$.

\section{Joint analysis of liver and muscle}

In both the muscle and liver, of the $\mathrm{DE}$ genes, 503 genes (Additional file 4: Table S4). were identified as being commonly DE between the LRFI and HRFI pigs . This is approximately half of the genes DE in the liver. 


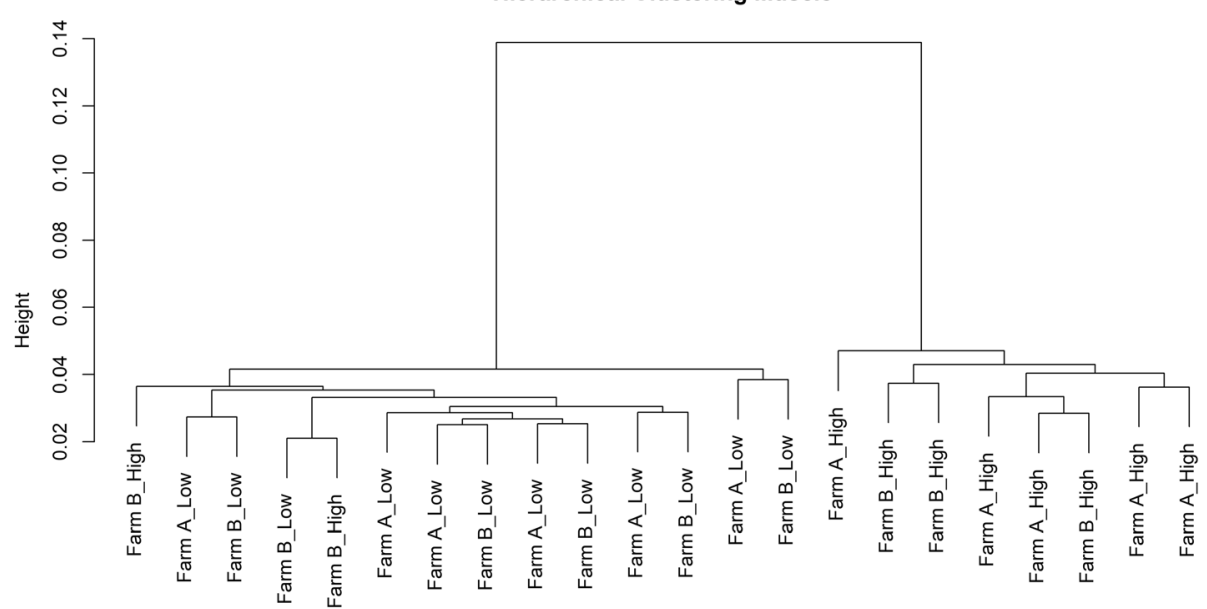

Fig. 1 Hierarchical clustering based on transcriptomic differences between the HRFI and LRFI pigs in muscle. The animals grouped by RFI status and not by farm of origin (Farm A and Farm B) in both tissues. The samples were clustered based on Ward method using 1 minus spearman correlation as distance

To aid in the understanding of the biological differences between RFI groups, GO term analysis of DE genes was conducted using a less stringent cutoff i.e. $q$-value $<0.10$, thus increasing the range of GO terms. These analyses were conducted separately for both the up regulated and down regulated genes. From the multi-tissue analysis of the DE genes in both muscle and liver, it was observed that the over represented GO terms from the up regulated genes in the LRFI vs. HRFI groups related to immune function and featured the terms, defense response and response to biotic stimulus (Table 3). The term defense response contained the majority of the $\mathrm{DE}$ genes $(A D A R$, CASP1, CUL1, CXCL10, DDX3X, DHX58, HERC5, IFI27, IFI44L, IFIT1, SGMS1, IFIT2, IFIT3, IFIT5 IFNGR1, IL1RAP, MX1, MX2, NLRP3, NR1D2, OASL, PARP9, PLGRKT, PSME4, RABGEf1, RORA, RSAD2, SP140, TRAF6, TRIM21, $X A F 1, Z B P 1)$. In contrast, genes relating to the term's protein targeting to membrane and extracellular matrix were under represented in the LRFI vs. HRFI pigs. The term protein targeting to membrane contains the genes (DMTN, GAS6, RPL (3, 18A), RPS (5, 11, 13, 19, 23) and SSR2). The term extracellular matrix organisation contains the genes

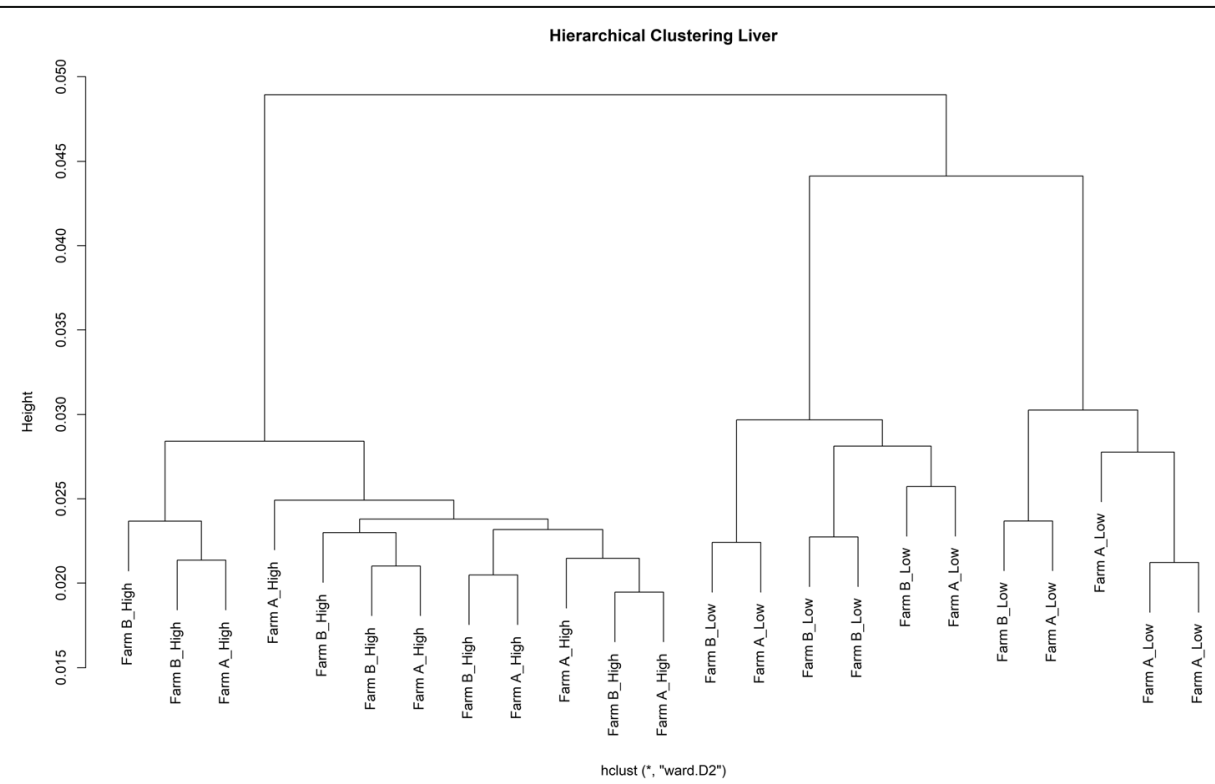

Fig. 2 Hierarchical clustering based on transcriptomic differences between the HRFI and LRFI pigs in liver. The animals grouped by RFI status and not by farm of origin (Farm A and Farm B) in both tissues. The samples were clustered based on Ward method using 1 minus spearman correlation as distance 
Table 2 Numbers of differentially expressed genes in LRFI compared to HRFI pigs in both Muscle and Liver

\begin{tabular}{|c|c|c|c|c|}
\hline \multirow{3}{*}{ Total $D E^{b}$ genes } & \multicolumn{2}{|l|}{ Muscle $^{a}$} & \multicolumn{2}{|l|}{ Liver $^{a}$} \\
\hline & \multicolumn{2}{|l|}{6463} & \multicolumn{2}{|l|}{964} \\
\hline & Up regulated & Down regulated & Up regulated & Down regulated \\
\hline Total genes & 3138 & 3325 & 467 & 496 \\
\hline$F C^{C} \leq 1.0$ & 1722 & 2465 & 365 & 384 \\
\hline$F C>1.0$ & 1381 & 851 & 77 & 100 \\
\hline$F C \geq 2.0$ & 35 & 10 & 25 & 12 \\
\hline
\end{tabular}

${ }^{a}$ LRFI vs. HRFI

${ }^{\mathrm{b}}$ Differentially expressed

c Fold change ratio

(CREB3L1, FMOD, GAS6, HTRA1, ITGA3, LTBP2, MMP15, MMP2, NOTCH1 OLFML2B, PDGFA TIMP).

\section{Differentially expressed genes in muscle}

Functional analysis using DAVID revealed an over-representation of genes related to the GO terms cellular protein modification process, RNA metabolic process, gene expression, cell cycle and regulation of nitrogen compound metabolic process with increased expression in the LRFI vs. HRFI groups. In contrast, terms such as mitochondrion organisation, establishment of protein localisation, cellular amide metabolic process, organonitrogen compound biosynthetic process, macromolecule localisation, cellular localisation, translation and generation of precursor metabolites and energy were over-represented in the down regulated gene list in the muscle of LRFI pigs (Table 4). The genes involved in a selected number of the GO terms are presented in Additional file 5: Table S2. Within the GO term mitochondrion organisation, the genes MFN2, ATG9A, MRPL38, NECTIN2, ATG4D and ATP2A1 are most significantly different between the RFI groups. Other genes of interest include: the $M R P$ genes $(2,4,10,11,16$, $18,24,28,38,44,46,48,52)$, the mitochondrial ribosomal genes MRPS (2, 6, 24, 26, 34), NDUF (A10, AF3, B7, B9, S2, S5, S6, S7, V1,), NMT1, TIMM (8B, 13, 17B, 22, 34, 40, 44), $U Q C C 2$ and $U Q C R 10$.

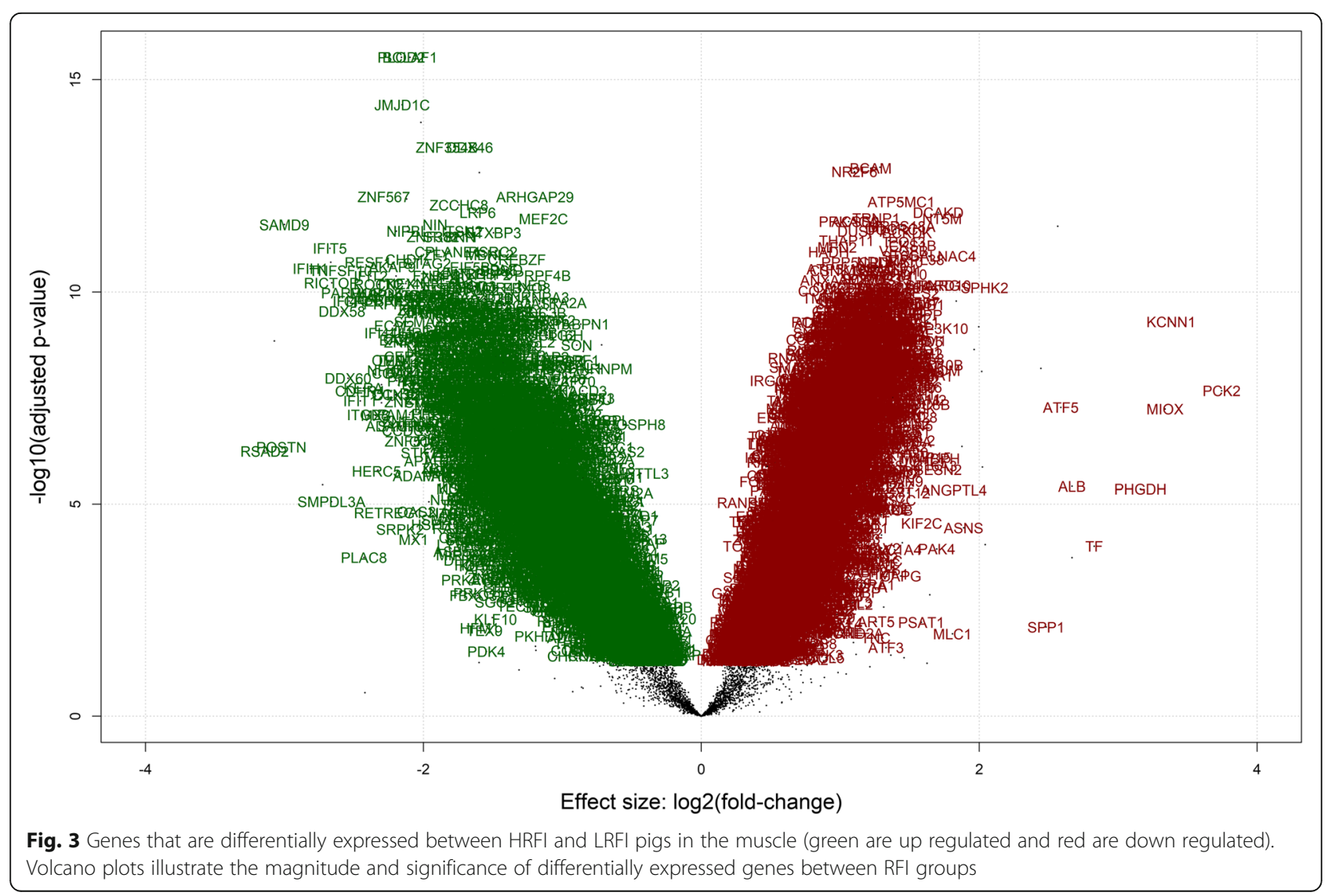




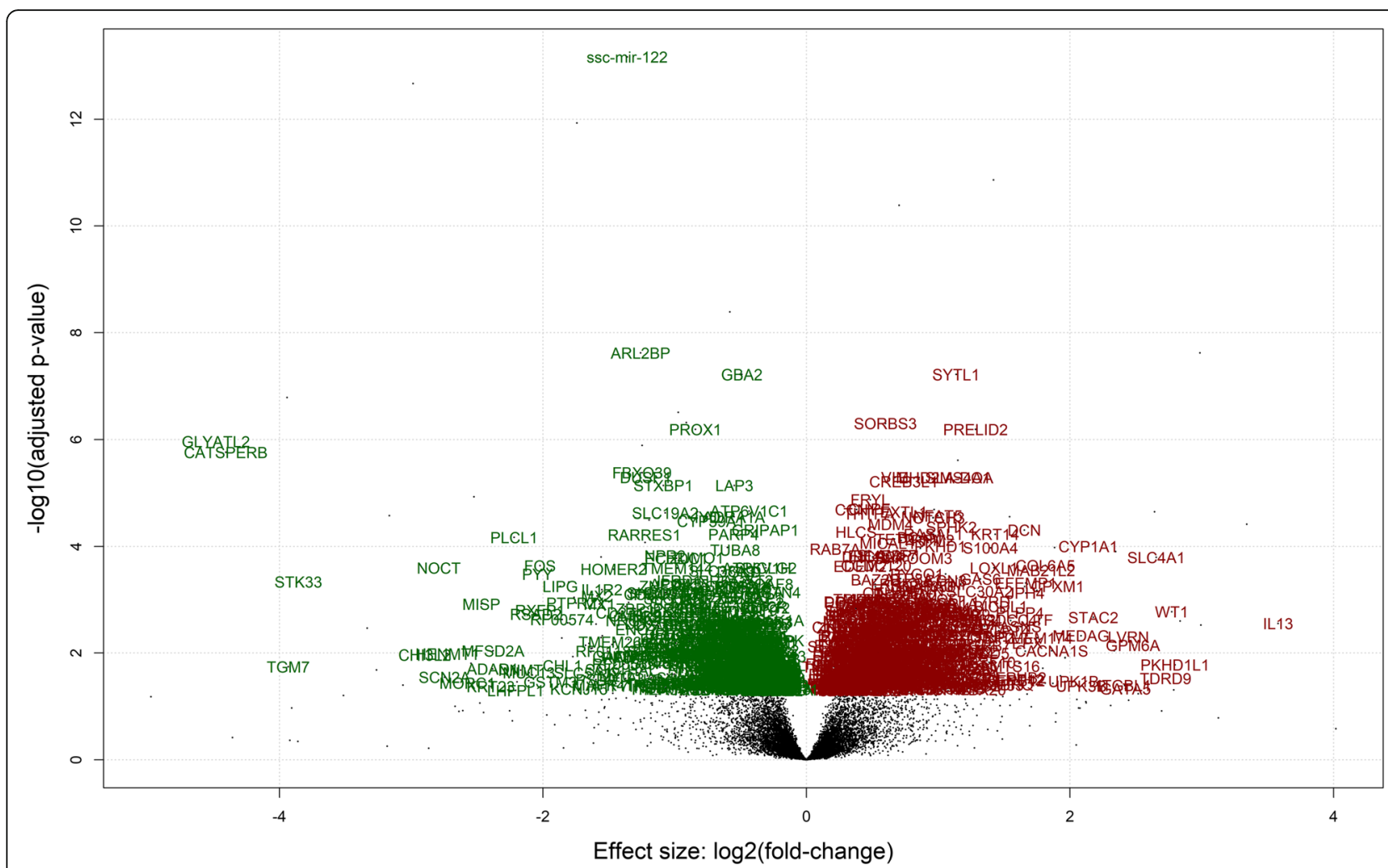

Fig. 4 Genes that are differentially expressed between HRFI and LRFI pigs in the liver (green are up regulated and red are down regulated). Volcano plots illustrate the magnitude and significance of differentially expressed genes between RFI groups

\section{Differentially expressed genes in liver}

Functional analysis using DAVID revealed an over-representation of genes with GO terms relating to: ribonucleoprotein complex biogenesis, RNA processing, defense response to virus, type 1 interferon signaling pathway, response to cytokine, RNA metabolic process, carboxylic catabolic process, defense response, lipid homeostasis and positive regulation of gene expression in the genes up regulated in the LRFI pigs (Table 5). The terms cell surface receptor signaling pathway, extracellular matrix organisation, extracellular structure organisation, biological adhesion, cell adhesion, regulation of multicellular organismal development, were down regulated in LRFI pigs. The genes involved in a selected number of the GO terms are presented in Additional file 6: Table S6.

\section{Discussion}

The current analysis provides novel findings in relation to the genes and processes associated with feed efficiency in pigs. We hypothesised, based on previous research, that genes associated with energy metabolism and immunity would be key influencers of feed efficiency. The results from this study partially support this hypothesis. In the joint analysis, variation in the expression of genes with the GO terms protein targeting to membrane, extracellular matrix organisation and a range of terms relating to immune function were $\mathrm{DE}$ between the LRFI and HRFI pigs. The analysis of the muscle tissue alone, highlighted genes with GO terms relating to RNA, protein synthesis and energy metabolism. The analysis of the liver tissue alone, highlighted genes with GO terms involved in lipid homeostasis. This analysis identifies a number of genes that are DE in both tissues and others which are tissue specific, all of which may be influencing RFI.

\section{Differentially expressed genes}

There was a notable difference in the number of $\mathrm{DE}$ genes in the muscle and liver with a 10-fold increase in genes expressed in the muscle compared to the liver. Interestingly, many of the GO terms in the muscle related to RNA and protein synthesis, supporting the increased transcriptomic activity in this tissue. A high proportion of $\mathrm{DE}$ genes had relatively subtle changes in gene expression with fold changes $<1$. This is consistent with other transcriptomic studies assessing variation in RFI in pigs, with the majority exhibiting small fold changes [7, 16-18]. While the reasons for the small fold changes in gene expression are unclear, Liu et al. [15] postulated that as pigs were not exposed to any external stimulus/challenge and were healthy, these 
expression levels therefore reflect the normal physiological range.

\section{Combined analysis of muscle and liver}

For the purposes of identifying robust markers of feed efficiency, we propose that the genes DE between LRFI and HRFI pigs in both muscle and liver are the most biologically significant. Indeed, 503 genes were commonly DE in the muscle and liver between the LRFI and HRFI pigs. Interestingly, this constituted approximately half of the genes expressed in the liver. The genes mainly grouped with three functions: protein targeting to membrane, extracellular matrix and immune function. A range of biologically relevant genes were DE within the term protein targeting to membrane (GAS6, RPL (19, 39), RPS $(5,11,13,18,19)$ and SSR2). GAS6 had lower expression in the LRFI group in comparison to the HRFI group. GAS6 which binds to the TYR03 receptor has previously been associated with cell differentiation in adipocytes and obesity in both humans and mice [19]. Interestingly in muscle, TYRO3 was DE between LRFI and HRFI pigs in the same direction to GAS6. It has previously been shown that LRFI pigs are leaner and have greater muscle content than their HRFI counterparts $[18,20,21]$. The changes in GAS6 and TYRO3 expression may influence body fat and increase efficiency in LRFI pigs. The majority of other genes in this term $(R P L$ $(3,18 \mathrm{~A}), \operatorname{RPS}(5,11,13,18,19)$ and $\operatorname{SSR} 2)$ are associated with ribosomal biogenesis and protein translation, functions which are important for cell growth and proliferation [22] and also the regulation of skeletal muscle mass [23]. All these genes had lower expression in the LRFI group in comparison to the HRFI group. Interestingly, an upstream regulator of ribosomal biogenesis, mTOR, had lower expression in the LRFI group in comparison to the HRFI group. mTOR has previously been attributed to the control of ribosomal biogenesis, a potential driver of the DE of the RPL and RPS genes and a factor impacting on differences in efficiency [23]. It could be inferred that the reduced expression of ribosomal proteins could contribute to energy reduction in the LRFI pigs.

The over-represented GO terms derived from the genes up regulated in the LRFI vs HRFI groups related to immune function and featured the terms, defence response and response to biotic stimulus. It was initially hypothesised that more efficient animals may be more susceptible to disease and stressors [24]. However, the more efficient LRFI pigs have been found to be more robust and better able to respond to infectious challenge [8]. With regard to RFI, immune-related data is highly variable and conflicting across transcriptomic studies. In some studies of both more efficient LRFI pigs and cattle there was also an up regulation of immune related genes $[25,26]$. However, more efficient pigs generally have a lower expression of genes related to immune function in non-challenged states $[16,17,27]$, an observation that is highly tissue dependent. The studies performed by Jégou et al [27] and Liu et al [16] differed from the current study, both in their findings and context, as the blood transcriptome, rather than that of the liver and muscle, was studied. However, the differing findings of Gondret et al [17] which included the analysis of muscle and liver, are more challenging to interpret. One potential explanation is that the pigs in this study were subjected to a pathogen/viral challenge in the form of vaccination or infection and hence a heightened immune response is reflected in this cohort. The pigs in this study received vaccination against post weaning multi-systemic wasting syndrome (PMWS). Potentially, the vaccination program

Table 3 Gene ontology terms overrepresented among differentially expressed genes in liver and muscle

\begin{tabular}{|c|c|c|c|}
\hline Term & Count & $\begin{array}{l}\text { B-H } \\
p \text {-value }\end{array}$ & \\
\hline \multicolumn{4}{|c|}{ GO terms overrepresented among DEGs with lower expression in the LRFI group vs HRFI group } \\
\hline $\begin{array}{l}\text { GO:0006612 protein } \\
\text { targeting to membrane }\end{array}$ & 10 & 2.59E-02 & DMTN, GAS6, RPL18A, RPL3, RPS5, RPS11, RPS13, RPS18, RPS19, SSR2 \\
\hline $\begin{array}{l}\text { GO:0030198 extracellular } \\
\text { matrix organization }\end{array}$ & 12 & $5.45 \mathrm{E}-02$ & CREB3L1, FMOD, GAS6, HTRA1, ITGA3, LTBP2, MMP15, MMP2, NOTCH1 OLFML2B, PDGFA TIMP \\
\hline \multicolumn{4}{|c|}{ GO terms overrepresented among DEGs with higher expression in the LRFI group vs HRFI group } \\
\hline $\begin{array}{l}\text { GO:0006952 defense } \\
\text { response }\end{array}$ & 32 & $9.10 \mathrm{E}-04$ & $\begin{array}{l}\text { ADAR, CASP1, CUL1, CXCL10, DDX3X, DHX58, HERC5, IFI27, IFI44L, IFIT1,SGMS1, IFIT2, IFIT3, IFIT5 } \\
\text { IFNGR1, ILIRAP, MX1, MX2, NLRP3, NR1D2,OASL, PARP9, PLGRKT, PSME4, RABGEF1, RORA, RSAD2, SP140, } \\
\text { TRAF6, TRIM21, XAF1, ZBP1, }\end{array}$ \\
\hline $\begin{array}{l}\text { GO:0009607 response to } \\
\text { biotic stimulus }\end{array}$ & 23 & $1.21 \mathrm{E}-03$ & $\begin{array}{l}\text { ABCA1, ADAR, CASP1, CCDC47, CXCL10, DDX3X, DHX58, HERC5, IFI44L, IFIT1, IFIT2, IFIT3, IFIT5, IFNGR1, } \\
\text { MX1, MX2, NLRP3, OASL, RSAD2, TRAF6, CMPK2, FMO1, SGMS1 }\end{array}$ \\
\hline $\begin{array}{l}\text { GO:0044764 multi-organism } \\
\text { cellular process }\end{array}$ & 24 & $1.42 \mathrm{E}-03$ & $\begin{array}{l}\text { ADAR, CXCL10, DDX3X, DHX58, HERC5, IFI44L, IFIT1, IFIT3, IFIT5, IFNGR1, MX1, MX2, NLRP3, NUP153, } \\
\text { OASL, PROX1, RSAD2, TOP1, TRIM21, ZMYND11, CFLAR, CUL1, IFIT2, USP7 }\end{array}$ \\
\hline $\begin{array}{l}\text { GO:0034097 response to } \\
\text { cytokine }\end{array}$ & 20 & $6.75 \mathrm{E}-03$ & $\begin{array}{l}\text { ADAR, CXCL10, IFI27, IFIT1, IFIT2, IFIT3, ILIRAP, MX1, MX2, OASL, PARP9, PSME4, RABGEF1, RORA, SGMS1, } \\
\text { TRAF6, TRIM21, XAF1, IFNGR1, RSAD2 }\end{array}$ \\
\hline
\end{tabular}


Table 4 Gene ontology terms overrepresented among differentially expressed genes in muscle

\begin{tabular}{|c|c|c|}
\hline Term & Count & B-H $p$-value \\
\hline \multicolumn{3}{|c|}{ GO terms overrepresented among DEGs with higher expression in the LRFI group vs HRFI } \\
\hline GO:0006464 cellular protein modification process & 765 & $1.75 \mathrm{E}-16$ \\
\hline GO:0016070 RNA metabolic process & 882 & $1.05 \mathrm{E}-12$ \\
\hline GO:0010467 gene expression & 987 & $1.05 \mathrm{E}-12$ \\
\hline GO:0007049 cell cycle & 371 & 4.46E-12 \\
\hline GO:0051171 regulation of nitrogen compound metabolic process & 831 & $2.62 \mathrm{E}-11$ \\
\hline GO:0034645 cellular macromolecule biosynthetic process & 921 & $2.43 \mathrm{E}-10$ \\
\hline GO:0006351 transcription, DNA-templated & 684 & 2.94E-09 \\
\hline GO:0070647 protein modification by small protein conjugation or removal & 235 & $2.69 \mathrm{E}-08$ \\
\hline GO:0051276 chromosome organisation & 256 & $5.62 \mathrm{E}-07$ \\
\hline GO:0006396 RNA processing & 206 & $5.83 \mathrm{E}-07$ \\
\hline \multicolumn{3}{|c|}{ GO terms overrepresented among DEGs with lower expression in the LRFI group vs HRFI } \\
\hline GO:0007005 mitochondrion organization & 204 & $1.31 \mathrm{E}-21$ \\
\hline GO:0045184 establishment of protein localisation & 445 & $1.64 \mathrm{E}-14$ \\
\hline GO:0043603 cellular amide metabolic process & 241 & $3.11 \mathrm{E}-11$ \\
\hline GO:1901566 organonitrogen compound biosynthetic process & 311 & $5.75 \mathrm{E}-11$ \\
\hline GO:0033036 macromolecule localisation & 562 & $1.57 \mathrm{E}-10$ \\
\hline GO:0051641 cellular localisation & 514 & $2.00 \mathrm{E}-10$ \\
\hline GO:0006412 translation & 167 & $5.88 \mathrm{E}-10$ \\
\hline GO:0006091 generation of precursor metabolites and energy & 112 & $1.15 \mathrm{E}-09$ \\
\hline GO:0009199 ribonucleoside triphosphate metabolic process & 87 & 4.70E-09 \\
\hline GO:0055114 oxidation-reduction process & 228 & 8.67E-09 \\
\hline
\end{tabular}

Table 5 Gene ontology terms overrepresented among differentially expressed genes in liver

\begin{tabular}{lll}
\hline Term & Count & B-H p-values \\
\hline GO terms overrepresented among DEGs with higher expression in the LRFI group vs HRFI group & 35 & $2.25 \mathrm{E}-04$ \\
GO:0022613 ribonucleoprotein complex biogenesis & 53 & $2.29 \mathrm{E}-04$ \\
GO:0006396 RNA processing & 22 & $5.93 \mathrm{E}-04$ \\
GO:0051607 defense response to virus & 13 & $5.99 \mathrm{E}-04$ \\
GO:0060337 type I interferon signalling pathway & 45 & 0.001367 \\
GO:0034097 response to cytokine & 161 & 0.004935 \\
GO:0016070 RNA metabolic process & 18 & 0.005979 \\
GO:0046395 carboxylic acid catabolic process & 67 & $5.93 \mathrm{E}-04$ \\
GO:0006952 defense response & 14 & 0.007424 \\
GO:0055088 lipid homeostasis & 71 & 0.012472 \\
GO:0010628 positive regulation of gene expression & & $1.86 \mathrm{E}-06$ \\
GO terms overrepresented among DEGs with lower expression in the LRFI group vs HRFI group & $1.91 \mathrm{E}-06$ \\
GO:0007166 cell surface receptor signalling pathway & 120 & $1.37 \mathrm{E}-06$ \\
GO:0030198 extracellular matrix organisation & 32 & $4.48 \mathrm{E}-06$ \\
GO:0043062 extracellular structure organisation & 32 & $6.60 \mathrm{E}-06$ \\
GO:0022610 biological adhesion & 85 & $6.00 \mathrm{E}-04$ \\
GO:0007155 cell adhesion & 84 & 78 \\
GO:2000026 regulation of multicellular organismal development & & \\
\hline
\end{tabular}


elicited an immune response in early life which persisted to slaughter, thus up regulating immune related genes and subsequently altering the ranking of the DE genes. Previously, a gene expression profile associated with viral immunity in adult sheep was attributed to vaccination in early life [28]. Interestingly, the expression pattern of several members of the gene families adenylate synthetase-like protein (OAS) and interferon-induced protein (IFIT) were common between the two studies. The fact that all animals were healthy prior to slaughter based on performance records, suggests that the increased expression of immune related genes did not have any negative effects on the animals in this study.

\section{Muscle}

A range of GO terms involved with RNA and protein synthesis had higher expression in the LRFI pigs compared to the HRFI group in muscle, with the most significant term being cellular protein modification process. This term is associated with the post translational modification of amino acids. The term relates to a number of genes (PRKGC1, MSTN, MAP3K1, ZNF451, IGF1) all of which could potentially be key to the differences in efficiency between RFI groups. In particular, the gene IGF1 has greater expression in the LRFI group compared to the HRFI pigs. Previously, IGF1 has been shown to correlate with RFI [29]. IGF1 is known to influence growth and development and therefore, could potentially be a key driver of feed efficiency [30]. Previously, several micro-RNAs were up regulated in LRFI pigs which the authors attributed to increased muscle growth and development through the IGF1/mTOR signalling pathway. Another gene of interest within this GO term is MSTN due to its functional role in muscle growth and development [31]. Similarly, MAP3K1 expression has been reported to be involved in tissue formation through protein synthesis [32]. The effect of changes in the expression of genes associated with muscle development in animals that have similar growth warrant further exploration. As the LRFI and HRFI pigs did not differ in ADG by having an increase in genes such as MAP3K1 these pigs may be more efficient at laying down muscle. Previously, genes associated with the term gene expression were identified as being significantly up regulated in adipose tissue, which is in agreement with the up regulation of genes related to this term in the liver of LRFI pigs [33]. Of the highlighted GO terms, the term translation elongation, a child term of translation was previously identified as being down regulated in LRFI pigs across a range of tissues, including liver, muscle perirenal and subcutaneous adipose tissue [17].

In addition, the GO term, mitochondrion organisation was most significantly down regulated in the LRFI compared to their HRFI counterparts in muscle. In combination with the GO term mitochondrion organisation, the terms generation of precursor metabolites and energy and oxidation reduction processes are terms which contain genes associated with mitochondrial function/energy metabolism. Mitochondria produce 95\% of energy within eukaryotic cells [34]. Changes in the expression of genes involved in the control of mitochondrial function are potentially key drivers of alterations in feed efficiency in pigs. Jing et al. [7] proposed that the LRFI pigs may be more efficient either as a result of reduced numbers of mitochondria or a down regulation of mitochondrial function. A number of genes; $C O X$, NDUF, SLC, and UQCR were up regulated in the HRFI compared to the LRFI pigs in this study, which is in agreement with previous studies $[6,7,33,35]$. PPARGC1B was also down regulated in the LRFI pigs in this study. PPARGC1B is a master regulator of mitochondrial biogenesis; the down regulation of this gene may be a key driver of differences in efficiency between LRFI and HRFI pigs. Increased expression of $P G C$ gene families such as PPARGC1B has been reported to increase energy expenditure and mitochondrial number [36]. The results suggest that ATP synthesis is potentially lower in the LRFI pigs than the HRFI pigs.

\section{Liver}

Genes involved in the GO term cell surface receptor signaling pathway were the most significantly up regulated genes in the liver of LRFI pigs relative to the HRFI group. This is a very broad GO term that has previously been identified as over-represented in the blood transcriptome of pigs from divergent selection lines [15]. The most significantly DE genes were VIM, CCNY, OGN, DCN and KRT19. These genes have a range of functions, including cell division, cell structure and bone formation. Genes associated with appetite control/feed intake/energy metabolism APOA4, $L E A P 2, R O R A, P P A R \alpha$ were identified within the GO term defense response. APOA4 was up regulated in the LRFI pigs, which was also observed in a different population of LRFI pigs with a similar genetic background to the pigs from Farm A in this study [17]. $A P O A 4$ has a role in satiety, is involved in the inhibition of appetite and has been attributed to the long-term regulation of feed intake [37, 38]. Similarly, $L E A P 2, R O R A$ and $P P A R \alpha$ were up regulated in the LRFI pigs. LEAP2 is an antagonist of the growth hormone secretagogue receptor $1 \mathrm{a}$ that binds to ghrelin, and is a key factor regulating systemic energy metabolism [39]. RORA is an upstream regulator of the $P P A R \alpha / R X R A$ complex. This complex acts as a transcription factor that activates genes involved in glycolysis/gluconeogenesis [40]. PPAR $\alpha$ has previously been associated with feed efficiency in pigs [33]. 
These genes are of interest as more efficient (LRFI) pigs have lower feed intake than HRFI pigs, suggesting differences in appetite regulation and energy metabolism [33, 41].

Genes involved in the GO term lipid homeostasis were over-expressed in the liver of LRFI pigs compared to the HRFI animals. Interesting up regulated genes associated with this term included LIPG, ACADL, ALSM1 and $A P O A 4$. LIPG plays a role in lipoprotein uptake and metabolism [42] and ACADL is involved in the beta-oxidation of fatty acids. ALSM1 is also involved in adipogenesis [43]. Lipogenesis in the liver generates fatty acids, which are esterified into triglycerides for storage in adipose tissue, oxidized in the liver or exported to other parts of the body as lipoproteins where they are used as an energy source and as membrane building components [44]. Based on this statement the increased expression of the genes associated with this GO term suggest that the more efficient (LRFI) pigs have improved capacity to metabolise fats, which may be an important driver of the improved efficiency in this group.

\section{Conclusion}

Overall the transcriptome data segregated based on RFI value rather than the genotype/farm of origin. Genes associated with GO terms protein targeting to membrane, extracellular matrix organisation and a range of terms relating to immune function were over represented in the DE genes of both liver and muscle. In the muscle, genes associated with GO terms involved in RNA, protein synthesis and energy metabolism were DE between efficient and inefficient pigs. In the liver, genes associated with lipid homeostasis were DE between the RFI groups. This suggests that there are common pathways across tissues as well as tissue specific pathways that contribute to differences in feed efficiency. These results highlight key genes APOA4, CPT1A, GAS6, IGF1, LEAP2, MAP3K1, MDUFB9, MSTN, mTOR, PPAR , PPARGC1B, PRKGC1, RORA, TYRO3 and ZNF451 as potential candidates for the identification of functional SNPs.

\section{Methods}

All experimental procedures described in this work were approved under University College Dublin animal research ethics committee (AREC-15-30-O'Doherty) and conducted under experimental license from the Department of Health in accordance with the cruelty to animal act 1876 and the European Communities (Amendments of Cruelty to Animal Act, 1876) Regulations (1994).

Two populations of pigs (Farms A and B) were utilised in this study. Pigs divergent in RFI were evaluated using a standard method [2]. Experimental conditions remained consistent between the two populations of pigs. The first population of pigs were the progeny of sows (Landrace $\mathrm{x}$ Large White) bred to Meatline boars (Farm A: Maxgro, Hermitage Pedigree Pigs, County Kilkenny Republic of Ireland) while the second population of pigs were progeny from sows (Landrace $\mathrm{x}$ Large White) bred to Meatline boars (Farm B: PIC Genetics, Line 37) and originated from a different breeding farm. The pigs in this study were vaccinated against post-weaning multi-systemic wasting syndrome (PMWS) and received the vaccination (CIRCOVAC) prior to weaning.

At weaning (day 28), pigs (144 and 140 pigs from Farms A and B, respectively) were transferred from their respective farms to UCD Lyons Research Farm (Newcastle, Co. Dublin, Ireland) and reared on standard commercial diets until slaughter and grouped in pens. Feed intake was recorded using single space computerized electronic feeders (Mastleistrungsprufing MLPRAP; Schauer Agrotronic AG, Sursee, Switzerland) according to the method of Varley et al [45]. To calculate RFI in population A, the BW of each animal was recorded on day 56 and subsequently on days 63, 70, 77, 84, 91, 98 and 105. Day 77 BW was used to calculate midtest metabolic BW (MBW). On day 105, a backfat (BF) scan was taken from all pigs in the area over the 10th rib. To calculate RFI in population B, the body weight of each animal was recorded on day 92 , and subsequently on day $99,106,113,120,127,134$ and 141. On day 133, bodyweight was used to calculate the mid-test metabolic body weight (MBW). On day 133, a backfat (BF) scan was taken from all pigs in the area over the 10th rib. In both populations, mid-test metabolic body weight was included and represented as $\mathrm{BW}^{0.60}$ which is representative of the maintenance requirement of the animal $[46,47]$. The RFI was the residual calculated using multiple regression model regressing average daily feed intake (ADFI) to average daily gain (ADG), BF and MBW. Standard deviations above and below the mean were used to group animals into HRFI (RFI $>0.5 \mathrm{SD}$ above the mean), and LRFI $(\mathrm{RFI}<-$ $0.5 \mathrm{SD})$.

\section{Animal selection and slaughter procedure}

In both Farms A and B, the 6 most efficient (LRFI) and 6 least efficient (HRFI) animals were selected for further analysis. Following fasting for a three-hour time period, the pigs were euthanized by lethal injection with Euthatal (Pentobarbitone Sodium BP; Merial Animal Limited) at a rate of $1 \mathrm{ml} / 2 \mathrm{~kg}$ body weight. Following slaughter, tissue sections $\left(1 \mathrm{~cm}^{2}\right)$ were sampled from the liver and Longissimus Dorsi muscle and stored in RNAlater (Ambion Inc., Austin, TX). Following an overnight incubation at $4{ }^{\circ} \mathrm{C}$, the RNAlater was removed and tissue was stored at $-70^{\circ} \mathrm{C}$ until RNA extraction. 


\section{RNA extraction}

Trizol Reagent (Sigma-Aldrich, Arklow, Ireland) was used to extract total RNA from liver $(50 \mathrm{mg})$ and muscle (100 mg). The GenElute Mammalian Total RNA Miniprep Kit (RTN70, Sigma-Aldrich) was used to further purify the crude RNA extract and included an on-column DNase step. The NanoDrop-ND1000 Spectrophotometer (Thermo Fisher Scientific Inc. MA, USA) was used to quantify RNA using the ratio of the absorbance at 260 and 280 $\mathrm{nm}$ with ratio above 1.8 being the acceptable cut-off. The Agilent RNA 6000 Nanochip bioanalyzer kit was used to assess RNA integrity with all samples having an RNA Integrity Number (RIN) above 8 (8.3 s.e. 0.59 ).

\section{RNA-sequencing}

The Institute of Molecular Medicine, University of Leeds, United Kingdom performed the library construction and RNA-seq. The RNA-seq libraries were constructed using the Illumina TruSeq RNA Sample Preparation Kit v2 (Illumina, San Diego, CA) according to the manufacturer's instructions. Adapters with unique barcodes were ligated to the end-polished cDNA fragments for each sample. The libraries were amplified by PCR, size selected and quantitated. The individual libraries were pooled with 24 libraries per pool. One hundred base paired-end sequencing was run on an Illumina HiSeq2000 platform with each pool ran on two lanes on a flow cell.

\section{Quality control read processing and alignment}

Read quality was checked by using FastQC (0.11.5). Adapters and low-quality reads were trimmed using the program Cutadapt (1.14) such that the average base quality was greater than 20 . Trimmed paired end reads were aligned to the pig reference genome Sscrofa 10.2 (version 87, Ensembl) using the alignment program HISAT2 (version 2.0.5), using the default settings $[48,49]$. SAM files were sorted using the sort procedure of SAMtools $[50,51]$. Using the program FeatureCounts (1.5.2), the sorted SAM files were used to generate read counts expressed per gene per library while the pig genome GTF file (version 10.2.87, Ensembl) as used as the genomic reference annotation file and default settings were applied [52].

\section{Statistical analysis of animal performance}

The performance data for both populations was analysed using the MIXED procedure (SAS Inst (version 9.4). Inc., Cary, NC) as a complete randomised design with RFI as the main effect and sow included as a random effect. Each population of animals was analysed separately. For all parameters examined, the individual pig was the experimental unit. The data was checked for normality using the UNIVARIATE procedure of SAS. All data presented in the tables are expressed as least squares means \pm standard error of the mean (SEM). Means were separated using the Tukey-Kramer method. The probability value, which denotes statistical significance was $P<0.05$.

\section{Statistical analysis of gene expression data}

The statistical language $\mathrm{R}$ was used for all statistical analyses of RNA-seq data (version 3.4.0). Count files produced from FeatureCounts were merged using the readDGE function within the $R$ package edge $R$ (version $3.18 .1)$ to produce a count table $[53,54]$. Hierarchical clustering was applied to identify outliers and based on this, one sample was removed from the liver dataset while two samples were excluded from the muscle data. Differential gene expression analysis was conducted using functions within the $\mathrm{R}$ package DESeq2 (version 1.16.1) [55]. As both populations grouped based on RFI, the gene expression data from Farms A and B were analysed together. The gene count data was normalised using the relative log expression method, based on "size factors" which accounts for RNA-seq library size differences. Dispersion estimates were also calculated. Pairwise comparisons of expression were then made between the LRFI and HRFI groups for each gene based on a negative binomial regression with farm included in the model. The output from DESeq2 included fold changes and associated $p$-values and adjusted $p$-values. A gene was defined as differentially expressed (DE) if the Benjamini Hochberg adjusted $p$-value was $\leq 0.05$. To account for variation between the populations, the farm of origin was included in the model. To facilitate further analysis, porcine Ensembl IDs were first uniquely mapped (one to one relationship only) to the more extensively annotated human orthologs, retrieved from the BioMart database [56]. All subsequent analyses were performed using these human orthologs and annotations.

The lists of DE genes were investigated for enrichment analysis of gene ontology (GO) terms for Biological Processes (BP), using the functional annotation tool of Database for Annotation, Visualization and Integrated Discovery (DAVID) bioinformatics resources [57]. The gene lists input in to DAVID were divided into over and under-represented groups in both liver and muscle. The background gene list used in the over and under-representation analysis included all genes with a one-to-one mapping with human orthologs for both liver and muscle. The GO term GOTERM_BP_FAT was selected to filter GO terms. The GOTERM_BP_FAT filters out broad GO terms based on the measured specificity of each term. For functional annotation analysis, GO terms were considered to be significant if they had a $q$-value of $(q<0.10)$. To identify commonalities between liver and muscle, the VENNY online tool [58] was used to compare the gene lists for up and down regulated genes in both tissues. 


\section{Additional files}

Additional file 1: Table S1. Sequencing information. This file contains raw sequence, read alignment and gene annotation information. (XLSX 13 kb) Additional file 2: Table S2. Differentially expressed genes in muscle. This table lists the genes that were differentially expressed in muscle. (XLSX $705 \mathrm{~kb}$ )

Additional file 3. Table S3. Differentially expressed genes in liver. This table lists the genes that were differentially expressed in liver. (XLSX 118 kb)

Additional file 4: Table S4. Common differentially expressed genes in liver \& muscle. This table lists the genes that were differentially expressed in both liver and muscle. (XLSX 27 kb)

Additional file 5: Table S5. Selected gene ontology terms in muscle. This table lists the genes involved in a selected number of gene ontology terms identified in muscle. (DOCX $23 \mathrm{~kb}$ )

Additional file 6: Table S6. Selected gene ontology terms in liver. This table lists the genes involved in a selected number of gene ontology terms identified in liver. (DOCX $16 \mathrm{~kb})$

\section{Abbreviations}

DAVID: Database for Annotation, Visualization and Integrated Discovery; GO: Gene ontology; HRFI: High residual feed intake; LRFI: Low residual feed intake; RFI: Residual feed intake

\section{Acknowledgements}

We wish to thank Dr. Marion Ryan for her help with editing this paper.

\section{Funding}

The authors would like to acknowledge funding from the Irish Department of Agriculture (DAFM) via the IdentiFEED project (13/S/519) which was provided to conduct this research. The funding body had no influence on the design of the study and collection, analysis, and interpretation of data and in writing the manuscript.

\section{Availability of data and materials}

RNA-Seq data has been submitted to ArrayExpress; accession E-MTAB-7435. All other relevant data is available within the manuscript or its accompanying supplementary material.

\section{Authors' contributions}

TS and JVOD designed this research project. SV performed the experiment. SV, KB analysed the data and SV, TS and JVOD wrote the paper. All authors have read and approved the manuscript prior to publication.

\section{Ethics approval and consent to participate}

All experimental procedures described in this work were approved under University College Dublin animal research ethics committee (AREC-15-30O'Doherty) and conducted under experimental license from the Department of Health in accordance with the cruelty to animal act 1876 and the European Communities (Amendments of Cruelty to Animal Act, 1876) Regulations (1994)

\section{Consent for publication}

Not applicable.

\section{Competing interests}

The authors declare that they have no competing interests.

\section{Publisher's Note}

Springer Nature remains neutral with regard to jurisdictional claims in published maps and institutional affiliations.

\section{Author details}

${ }^{1}$ School of Veterinary Medicine, University College Dublin, Belfield, Dublin 4 Ireland. ${ }^{2}$ School of Agriculture \& Food Science, University College Dublin, Belfield, Dublin 4, Ireland.
Received: 12 November 2018 Accepted: 26 April 2019

Published online: 06 June 2019

\section{References}

1. Koch RM, Swiger LA, Chambers D, Gregory KE. Efficiency of feed use in beef cattle. J Anim Sci. 1963;22(2):486-94.

2. Vigors S, Sweeney T, O'Shea CJ, Kelly AK, O'Doherty JV. Pigs that are divergent in feed efficiency, differ in intestinal enzyme and nutrient transporter gene expression, nutrient digestibility and microbial activity. Animal. 2016;10(11):1848-55.

3. Patience JF, Rossoni-Serao MC, Gutierrez NA. A review of feed efficiency in swine: biology and application. J Anim Sci Biotechnol. 2015;6(1):33.

4. Rakhshandeh A, Dekkers JC, Kerr BJ, Weber TE, English J, Gabler NK. Effect of immune system stimulation and divergent selection for residual feed intake on digestive capacity of the small intestine in growing pigs. J Anim Sci. 2012;90(Suppl 4):233-5.

5. Grubbs JK, Huff-Lonergan E, Gabler NK, JC MD, Lonergan SM. Liver and skeletal muscle mitochondria proteomes are altered in pigs divergently selected for residual feed intake. J Anim Sci. 2014;92(5):1995-2007.

6. Fu L, Xu Y, Hou Y, Qi X, Zhou L, Liu H, Luan Y, Jing L, Miao Y, Zhao S, et al. Proteomic analysis indicates that mitochondrial energy metabolism in skeletal muscle tissue is negatively correlated with feed efficiency in pigs. Sci Rep. 2017;7:45291.

7. Jing L, Hou Y, Wu H, Miao Y, Li X, Cao J, Brameld JM, Parr T, Zhao S. Transcriptome analysis of mRNA and miRNA in skeletal muscle indicates an important network for differential residual feed intake in pigs. Sci Rep. 2015; 5:11953

8. Vigors S, O'Doherty JV, Kelly AK, O'Shea CJ, Sweeney T. The effect of divergence in feed efficiency on the intestinal microbiota and the intestinal immune response in both unchallenged and lipopolysaccharide challenged Ileal and colonic explants. PLoS One. 2016;11(2):e0148145.

9. Mani V, Harris AJ, Keating AF, Weber TE, Dekkers JCM, Gabler NK. Intestinal integrity, endotoxin transport and detoxification in pigs divergently selected for residual feed intake. J Anim Sci. 2013;91(5):2141-50.

10. Hayes BJ, Lewin HA, Goddard ME. The future of livestock breeding: genomic selection for efficiency, reduced emissions intensity, and adaptation. Trends Genet. 2013;29(4):206-14.

11. Saintilan R, Mérour I, Brossard L, Tribout T, Dourmad JY, Sellier P, Bidanel J, van Milgen J, Gilbert H. Genetics of residual feed intake in growing pigs: relationships with production traits, and nitrogen and phosphorus excretion traits. J Anim Sci. 2013:91(6):2542-54.

12. Zhang C, Kemp RA, Stothard P, Wang Z, Boddicker N, Krivushin K, Dekkers J, Plastow G. Genomic evaluation of feed efficiency component traits in Duroc pigs using $80 \mathrm{~K}$, 650K and whole-genome sequence variants. Genet Sel Evol. 2018:50(1):14

13. Onteru SK, Gorbach DM, Young JM, Garrick DJ, Dekkers JC, Rothschild MF. Whole genome association studies of residual feed intake and related traits in the pig. PLoS One. 2013:8(6):e61756.

14. Do DN, Ostersen T, Strathe AB, Mark T, Jensen J, Kadarmideen HN. Genomewide association and systems genetic analyses of residual feed intake, daily feed consumption, backfat and weight gain in pigs. BMC Genet. 2014;15:27.

15. Reyer H, Oster M, Magowan E, Dannenberger D, Ponsuksili S, Wimmers K. Strategies towards improved feed efficiency in pigs comprise molecular shifts in hepatic lipid and carbohydrate metabolism. Int J Mol Sci. 2017: 18(8):1674.

16. Liu H, Nguyen YT, Nettleton D, Dekkers JCM, Tuggle CK. Post-weaning blood transcriptomic differences between Yorkshire pigs divergently selected for residual feed intake. BMC Genomics. 2016;17:73.

17. Gondret F, Vincent A, Houée-Bigot M, Siegel A, Lagarrigue S, Causeur D, Gilbert H, Louveau I. A transcriptome multi-tissue analysis identifies biological pathways and genes associated with variations in feed efficiency of growing pigs. BMC Genomics. 2017;18:244.

18. Horodyska J, Oster M, Reyer H, Mullen AM, Lawlor PG, Wimmers K, Hamill RM. Analysis of meat quality traits and gene expression profiling of pigs divergent in residual feed intake. Meat Sci. 2018;137:265-74.

19. Wu K-S, Hung Y-J, Lee C-H, Hsiao F-C, Hsieh P-S. The involvement of GAS6 signaling in the development of obesity and associated inflammation. Int J Endocrinol. 2015;2015:202513.

20. Lefaucheur L, Lebret B, Ecolan P, Louveau I, Damon M, Prunier A, Billon Y, Sellier $P$, Gilbert H. Muscle characteristics and meat quality traits are affected 
by divergent selection on residual feed intake in pigs. J Anim Sci. 2011; 89(4):996-1010.

21. Faure J, Lefaucheur L, Bonhomme N, Ecolan P, Meteau K, Coustard SM, Kouba M, Gilbert H, Lebret B. Consequences of divergent selection for residual feed intake in pigs on muscle energy metabolism and meat quality. Meat Sci. 2013:93(1):37-45.

22. Zhou X, Liao W-J, Liao J-M, Liao P, Lu H. Ribosomal proteins: functions beyond the ribosome. J Mol Cell Biol. 2015;7(2):92-104.

23. Chaillou T, Kirby TJ, McCarthy J. Ribosome biogenesis: emerging evidence for a central role in the regulation of skeletal muscle mass. J Cell Physiol. 2014;229(11):1584-94.

24. Rauw W, Kanis E, Noordhuizen-Stassen E, Grommers F. Undesirable side effects of selection for high production efficiency in farm animals: a review. Livest Prod Sci. 1998;56(1):15-33.

25. Weber KL, Welly BT, Van Eenennaam AL, Young AE, Porto-Neto LR, Reverter A, Rincon G. Identification of gene networks for residual feed intake in Angus cattle using genomic prediction and RNA-seq. PLoS One. 2016;11(3): e0152274

26. Horodyska J, Wimmers K, Reyer H, Trakooljul N, Mullen AM, Lawlor PG, Hamill RM. RNA-seq of muscle from pigs divergent in feed efficiency and product quality identifies differences in immune response, growth, and macronutrient and connective tissue metabolism. BMC Genomics. 2018; 19(1):791.

27. Jégou M, Gondret F, Vincent A, Tréfeu C, Gilbert H, Louveau I. Whole blood transcriptomics is relevant to identify molecular changes in response to genetic selection for feed efficiency and nutritional status in the pig. PLoS One. 2016;11(1):e0146550.

28. Ahmed AM, Good B, Hanrahan JP, McGettigan P, Browne J, Keane OM, Bahar B, Mehta J, Markey B, Lohan A, et al. Variation in the ovine Abomasal lymph node transcriptome between breeds known to differ in resistance to the gastrointestinal nematode. PLoS One. 2015;10(5):e0124823.

29. Bunter KL, Cai W, Johnston DJ, Dekkers JC. Selection to reduce residual feed intake in pigs produces a correlated response in juvenile insulin-like growth factor-I concentration. J Anim Sci. 2010;88(6):1973-81.

30. Hossner KL, McCusker RH, Dodson MV. Insulin-like growth factors and their binding proteins in domestic animals. Anim Sci. 2010;64:1):1-15.

31. Stinckens A, Luyten T, Bijttebier J, Van den Maagdenberg K, Dieltiens D, Janssens S, De Smet S, Georges M, Buys N. Characterization of the complete porcine MSTN gene and expression levels in pig breeds differing in muscularity. Anim Genet. 2008;39(6):586-96.

32. Naraballobh W, Trakooljul N, Murani E, Brunner R, Krischek C, Janisch S, Wicke M, Ponsuksili S, Wimmers K. Transient shifts of incubation temperature reveal immediate and long-term transcriptional response in chicken breast muscle underpinning resilience and phenotypic plasticity. PLoS One. 2016;11(9):e0162485.

33. Lkhagvadorj S, Qu L, Cai W, Couture OP, Barb CR, Hausman GJ, Nettleton D, Anderson LL, Dekkers JC, Tuggle CK. Gene expression profiling of the shortterm adaptive response to acute caloric restriction in liver and adipose tissues of pigs differing in feed efficiency. Am J Physiol. 2010;298(2):R494-507.

34. Tzameli I. The evolving role of mitochondria in metabolism. Trends in Endocrinology \& Metabolism. 2012;23(9):417-9.

35. Vincent A, Louveau I, Gondret F, Trefeu C, Gilbert H, Lefaucheur L. Divergent selection for residual feed intake affects the transcriptomic and proteomic profiles of pig skeletal muscle. J Anim Sci. 2015;93(6):2745-58.

36. Lehman JJ, Barger PM, Kovacs A, Saffitz JE, Medeiros DM, Kelly DP. Peroxisome proliferator-activated receptor $y$ coactivator-1 promotes cardiac mitochondrial biogenesis. J Clin Invest. 2000;106(7):847-56.

37. Woods SC. Dietary synergies in appetite control: distal gastrointestinal tract. Obesity. 2006;14(S7):171S-8S.

38. Shen L, Tso P, Woods SC, Sakai RR, Davidson WS, Liu M. Hypothalamic apolipoprotein A-IV is regulated by leptin. Endocrinology. 2007;148(6):2681-9.

39. Al-Massadi O, Müller T, Tschöp M, Diéguez C, Nogueiras R. Ghrelin and LEAP-2: rivals in energy metabolism. Trends Pharmacol Sci. 2018;39(8):685-94.

40. Soltis AR, Motola S, Vernia S, Ng CW, Kennedy NJ, Dalin S, Matthews BJ, Davis RJ, Fraenkel E. Hyper- and hypo- nutrition studies of the hepatic transcriptome and epigenome suggest that PPARalpha regulates anaerobic glycolysis. Sci Rep. 2017;7(1):174.

41. Vigors S, O'Doherty JV, Fahey AG, O'Shea CJ, Sweeney T. The relationship between feed efficiency and the expression of genes associated with appetite control in the hypothalamus and intestine of pigs. J Anim Sci. 2016;94(supplement3):222-5.
42. Holmes RS, VandeBerg JL, Cox LA. Vertebrate endothelial lipase: comparative studies of an ancient gene and Protein in vertebrate evolution Genetica. 2011;139(3):291-304.

43. Romano S, Milan G, Veronese C, Collin GB, Marshall JD, Centobene C, Favaretto F, Dal Pra C, Scarda A, Leandri S. Regulation of Alström syndrome gene expression during adipogenesis and its relationship with fat cell insulin sensitivity. Int J Mol Med. 2008;21(6):731-6.

44. Mukiibi R, Vinsky M, Keogh KA, Fitzsimmons C, Stothard P, Waters SM, Li C. Transcriptome analyses reveal reduced hepatic lipid synthesis and accumulation in more feed efficient beef cattle. Sci Rep. 2018;8(1):7303.

45. Varley PF, Flynn B, Callan JJ, O'Doherty JV. Effect of phytase level in a low phosphorus diet on performance and bone development in weaner pigs and the subsequent effect on finisher pig bone development. Livest Sci. 2011;138(1-3):152-8.

46. NRC. Nutrient requirements of swine, vol. 10th ed. Washington, D.C. 20418: National Academy Press; 2012.

47. Kil DY, Kim BG, Stein HH. Feed energy evaluation for growing pigs. Asian Australas J Anim Sci. 2013;26(9):1205-17.

48. Kim D, Langmead B, Salzberg SL. HISAT: a fast spliced aligner with low memory requirements. Nat Methods. 2015;12(4):357-60.

49. Pertea M, Kim D, Pertea GM, Leek JT, Salzberg SL. Transcript-level expression analysis of RNA-seq experiments with HISAT, StringTie and Ballgown. Nat Protoc. 2016;11(9):1650-67.

50. Li H, Handsaker B, Wysoker A, Fennell T, Ruan J, Homer N, Marth G, Abecasis $G$, Durbin R. The sequence alignment/map format and SAMtools. Bioinformatics. 2009;25(16):2078-9.

51. Li H. A statistical framework for SNP calling, mutation discovery, association mapping and population genetical parameter estimation from sequencing data. Bioinformatics. 2011;27(21):2987-93.

52. Liao Y, Smyth GK, Shi W. featureCounts: an efficient general purpose program for assigning sequence reads to genomic features. Bioinformatics. 2014;30(7):923-30

53. Robinson MD, McCarthy DJ, Smyth GK. edgeR: a Bioconductor package for differential expression analysis of digital gene expression data. Bioinformatics. 2010;26(1):139-40.

54. McCarthy DJ, Chen Y, Smyth GK. Differential expression analysis of multifactor RNA-Seq experiments with respect to biological variation. Nucleic Acids Res. 2012;40(10):4288-97.

55. Love Ml, Huber W, Anders S. Moderated estimation of fold change and dispersion for RNA-seq data with DESeq2. Genome Biol. 2014;15(12):550

56. Kasprzyk A. BioMart: driving a paradigm change in biological data management. Database (Oxford). 2011;2011:bar049.

57. Huang d W, Sherman BT, Lempicki RA. Systematic and integrative analysis of large gene lists using DAVID bioinformatics resources. Nat Protoc. 2009; 4(1):44-57.

58. Öliveros JC: VENNY. An interactive tool for comparing lists with Venn Diagrams. http://bioinfogp.cnb.csic.es/tools/venny/index.html. 2017.

\section{Ready to submit your research? Choose BMC and benefit from:}

- fast, convenient online submission

- thorough peer review by experienced researchers in your field

- rapid publication on acceptance

- support for research data, including large and complex data types

- gold Open Access which fosters wider collaboration and increased citations

- maximum visibility for your research: over $100 \mathrm{M}$ website views per year

At $\mathrm{BMC}$, research is always in progress.

Learn more biomedcentral.com/submission 